\title{
CONNECTIVE TISSUE DISEASES
}

\section{Blood vessel microenvironment sustains cell renewal in Dupuytren contracture nodules}

Small blood vessels associated with proliferative cells in Dupuytren contracture nodules have an important role in the pathogenesis of the disease, according to a study published in Arthritis Research \& Therapy. Viil et al. suggest the presence of certain growth factors and the composition of the extracellular matrix (ECM) provide a microenvironment conducive to the sustained proliferation of contractile myofibroblasts-a putative stem cell niche.

Although the proliferation of myofibroblasts is widely accepted to be central to the formation of the nodules and fibrous chords that characterize Dupuytren contracture tissue, the underlying cellular and molecular mechanisms remain largely unknown. "We believe that uncovering such mechanisms might not just help to treat this disease in a less invasive manner, but also would give us valuable knowledge about the mechanisms that initiate, maintain and terminate fibroblast regeneration," explains the study's corresponding author, Viljar Jaks.

4 ...proliferative cells are concentrated in and immediately around the blood vessels, chiefly in the myofibroblast layer... 77

Immunofluorescence confocal microscopy of fibrotic palmar fascia tissue from patients with Dupuytren contracture revealed that proliferative cells are concentrated in and immediately around the blood vessels, chiefly in the myofibroblast layer of the blood vessel walls. Activated AKT signalling, a pro-proliferative signalling pathway, was detected in the blood vessels and sweat glands of the tissue. Also expressed in the tissue were several activators of AKT signalling, including basic fibroblast growth factor (bFGF) in the endothelium of the blood vessels, insulin-like growth factor 2 (IGF-2) uniformly throughout the nodular tissue, and connective tissue growth factor (CTGF) in sweat glands in the vicinity of the nodule. The ECM was enriched for laminins 511 and 521, which colocalized with collagen IV in the small blood vessels.

The investigators will next attempt to use the components of the putative stem cell niche to recreate the sustained cell proliferation of Dupuytren contracture ex vivo. "We are planning to test whether and how the growth and identity of normal fibroblasts is affected by their cultivation on laminin 511/521-enriched substrates and in the presence of growth factors identified by us and others," Jaks concludes.

\section{Sarah Onuora}

Original article Viil, J. et al. Laminin-rich blood vessels display activated growth factor signaling and act as the proliferation centers in Dupuytren's contracture. Arthritis Res. Ther. 17, 144 (2015) 